\title{
Quality of life assessment by applying EORTC questionnaires to rectal cancer patients after surgery and neoadjuvant and adjuvant treatment
}

\author{
Juan Ignacio Arraras ${ }^{1,2}$, Javier Suárez ${ }^{3}$, Fernando Arias-de-la-Vega ${ }^{1}$, Ruth Vera ${ }^{2}$, Berta Ibáñez $^{4}$, \\ Gemma Asin ${ }^{1}$, Antonio Viudez ${ }^{2}$, Uxue Zarandona ${ }^{1,2}$, Mikel Rico ${ }^{1}$ and Irene Hernández ${ }^{2}$
}

Departments of ${ }^{1}$ Radiotherapeutic Oncology, ${ }^{2}$ Medical Oncology and ${ }^{3}$ Surgery. Complejo Hospitalario de Navarra. Pamplona, Navarra. Spain. ${ }^{4}$ Centro de Investigación Biomédica. Pamplona, Navarra. Spain

\begin{abstract}
Background: Quality of Life (QoL) is a key element in rectal cancer $(\mathrm{RC})$ patients.

Aims: this study assesses QoL in a sample of $\mathrm{RC}$ patients in their treatment follow-up period, and compares surgery modalities.

Patients and methods: eighty four locally advanced RC patients who had received surgery and neoadjuvant chemoradiotherapy were included in the study. Of these, 70 had adjuvant chemotherapy. All patients completed the EORTC QLQ-C30 and the QLQ-CR29 once at least one year after completion of their treatment. Low anterior resection (LAR) patients also completed a Functional Evaluation questionnaire.

Results: QoL scores in the EORTC questionnaires for the sample as a whole were high in most dimensions, in line with the general population's QoL values, although moderate limitations ( $>30$ points) were observed in urinary frequency, flatulence, impotence and sexual function. The scores for the Functional Evaluation were adequate (mean combined bowel function score of 18.2).

LAR patients had a higher stool frequency than those with abdominoperineal resection (APR; $p<0.001$ ). No differences in body image were found amongst LAR and APR patients.

LAR patients with a lower anastomosis had higher faecal incontinence $(p=0.02)$, whereas those with a reservoir had better emotional functioning $(p=0.04)$ and higher faecal incontinence $(p=$ 0.03).

Conclusions: QoL scores and functional evaluation indicated patients had adapted to their disease and treatment. The few differences in QoL found between surgery modalities are in line with other recent studies and in contrast with earlier ones that suggested a lower QoL in APR patients.
\end{abstract}

Key words: Cancer. Quality of life. Surgery. Rectal. Chemoradiotherapy.

Received: $15-01-2013$

Accepted: 07-05-2013

Correspondence: Juan Ignacio Arraras. Department of Oncology. Complejo Hospitalario de Navarra. C/ Irunlarrea, 3. 31008 Pamplona, Navarra. Spain e-mail: jiarraras@correo.cop.es
Arraras JI, Suárez J, Arias-de-la-Vega F, Vera R, Ibáñez B, Asin G, Viudez A, Zarandona U, Rico M, Hernández I. Quality of Life assessment by applying EORTC questionnaires to rectal cancer patients after surgery and neoadjuvant and adjuvant treatment. Rev Esp Enferm Dig 2013;105:255-261.

\section{INTRODUCTION}

Rectal cancer (RC) and its treatments can influence different dimensions of patients' quality of life (QoL), especially social and emotional aspects, such as body image and sexuality, and medical aspects like bowel movements or fatigue $(1,2)$. Such aspects can have influence in the decision about to perform a low anterior resection (LAR) or abdominoperineal resection (APR) procedure.

It was initially assumed that a permanent stoma should be avoided to improve patients' QoL, mainly due to an expected higher psychological burden. However, although several QoL studies have been undertaken to compare LAR and APR, few differences, in only specific areas, have been found $(3,4)$. More research on this topic is therefore needed to be able to reach more solid conclusions. In this respect, Cornish et al. (5) have suggested to undertake more studies with a follow-up period longer than one year.

One area in which research on surgery modalities has concentrated is body image, although with differing results. Kasparek et al. (6) found differences between LAR and APR in men but not in women. A further area of research is the QoL of patients with different anastomotic heights. There is a general tendency to consider that the closer the anastomosis is to the anal verge, the worse the surgical and functional outcome is likely to be $(7,8)$. However, Guren et al. (9) consider that there are no clear results regarding the specific anastomotic height where QL starts to deteriorate.

The influence of a J-pouch reservoir on QoL is another key aspect. Thus, it has been reported that a colonic J-Pouch results 
in a lower defecation urgency and reduction in stool frequency than straight coloanal anastomosis (7). More research on the attitude towards anastomosis, the presence of a reservoir, and on the QoL areas affected could therefore be useful in order to be able to reach more solid conclusions. The role of neoadjuvant treatments on QoL is also important (10).

The aims of the present study were to study QoL in RC patients who had received a combined treatment and were in the follow-up period for more than one year, to compare the QoL of RC patients who had received LAR or APR, and to contribute to the study of the influence of anastomotic height and the presence of a reservoir on patients' QoL. Our hypotheses were that QoL scores for the sample as a whole should be high, with moderate limitations in specific disease and treatment areas. A small number of differences, mainly concerning gastrointestinal symptoms, were expected, and would favour LAR patients, those with a reservoir and with a higher anastomosis.

\section{PATIENTS AND METHODS}

\section{Participants}

A consecutive sample of stage II-III RC patients (TNM classification $5^{\text {th }}$ version) was recruited. Patients had undergone surgery six to eight weeks after receiving neoadjuvant treatment and were disease-free at the time of assessment (at least one year from their treatment end, including ileostomy closing). Patients received one of two surgical modalities: a) Colorectal or coloanal anastomosis (with or without a temporary ileostomy) after LAR; these patients might also have a colonic reservoir; and b) definitive colostomy after an abdominoperineal resection (APR).

All patients had received neoadjuvant radiotherapy over five weeks at a dose of 4,500 cGy. The tumour area was then over-printed to a total of 5,040 cGy. They received concomitant fluoropyridine-based chemotherapy and could also have adjuvant chemotherapy on the basis of their performance status, response to neoadjuvant treatment, and disease stage.

Follow-up schema included digital examination and CEA level determination every three months for the first and second years, and then at six months interval during three years. Chest $\mathrm{x}$-ray and abdomen-pelvic TC were performed yearly and periodic colonoscopy on the basis of the risk of each patient.

\section{Measures}

All patients completed the EORTC questionnaires QLQC30 version 3.0) (11) and QLQ-CR29 (12), which our group had validated for use in our country $(13,14)$, and had been translated into Spanish following the translation procedure of the EORTC QoL Study Group (15). The structure of these questionnaires is shown in table I. QLQ-C30 evaluates areas common to different tumour sites and treatments, whereas QLQ-CR29 evaluates the areas associated with colorectal cancer and its treatments. The EORTC QLQ-CR29 is an update of the EORTC QLQ-C38, which it replaces (16). These two versions share two similar scales whereas other areas have a related or different content. The QLQ-CR29 allows for a better comparison between surgical modalities than the QLQ-C38 by including five separate scales for patients with or without stoma which can then be compared.

\section{Functional evaluation}

Patients with anastomosis were evaluated using a modified version of the questionnaire created by Hallböök et al. $(17,18)$ that includes 12 items. The mean number of bowel movements in 24 hours (based on the data for a week) was calculated, and the degree of faecal incontinence was measured using a composite scoring system (range 0 minimum to 18 maximum). The other parameters had four answer categories. The answers to these ten questions were categorised into two groups (good or poor function) for the statistical analysis. A combined bowel function score based on four variables, namely mean number of bowel move-

Table I. Contents of the EORTC General Questionnaire QLQ-C30 and Colorectal Cancer Module QLQ-CR29

\begin{tabular}{ll}
\hline EORTC QLQ-C30 & General questionnaire \\
Functioning scales $^{\mathrm{a}}$ & Physical, role, cognitive, \\
& emotional, social and global \\
& quality of life \\
Symptom scales and items & Fatigue, nausea and vomiting, \\
& pain, dyspnoea, sleep \\
& disturbance, appetite loss, \\
& constipation, diarrhoea, financial \\
& impact
\end{tabular}

EORTC QLQ-CR29

Functioning scales and items ${ }^{\mathrm{a}}$ Body image, anxiety, weight, sexual function men and women $* * *$

Symptom scales and items ${ }^{b} \quad$ Urinary frequency, blood and mucus in stool, stool frequency**, urinary incontinence, dysuria, abdominal pain, buttock pain, bloated feeling, dry mouth, hair loss, trouble with taste, flatulence**, faecal incontinence ${ }^{* *}$, sore skin**, embarrassed by bowel movement**, stoma care problems, impotence, dyspareunia

Higher scores in areas (a) represent better functioning, whereas higher scores in areas (b) indicate a higher level of symptoms. **Each of these areas contains different questions for patients with and without stoma that can be compared. ${ }^{* * *}$ There are separate items to evaluate sexual function for men and women. 
Table II. Characteristics of the sample

\begin{tabular}{ll}
\hline Area & $n(\%)$ \\
\hline Age** & Mean 65.2 SD 9.5 \\
range 44-82 & \\
Civil status & \\
Single & $2(2.5)$ \\
Married & $76(90.4)$ \\
Widow(er) & $6(7.1)$ \\
Gender & \\
Female & $28(33.3)$ \\
Male & $56(66.7)$ \\
Limiting comorbidity & $36(42.9)$ \\
Karnofsky* & Mean 86.2 SD 16.7 \\
Adjuvant treatment & \\
QT & $70(83.3)$ \\
Surgical modality & \\
LAR & $26(30.9)$ \\
LAR + reservoir & $34(40.5)$. \\
APR & $24(28.6)$ \\
Definitive stoma & $24(28.6)$ \\
Temporary ileostomy & $46(76.7)$ \\
Distance between anastomosis and & \\
anal verge & \\
2 to 5 & \\
6 to 12 & $38(63.3)$ \\
& $22(36.7)$ \\
& \\
&
\end{tabular}

**Mean and standard deviation are presented for the areas age and Karnofsky.

ments in 24 hours, composite faecal incontinence score, ability to defer defecation for 30 minutes, and ability to empty the bowel in less than 15 minutes, was also created. For each variable, patients were ranked from the most to the least favourable, and the mean of the four ranks obtained was considered to be an indicator of bowel function.

Sociodemographic and clinical data were taken from clinical records and performance status was assessed by the physician (Karnofsky scale, KPS) (19). QoL questionnaires with less than $70 \%$ of the items answered were excluded.

\section{Data collection procedures}

Patients were interviewed and invited to participate in the study. They were given oral and written information concerning the research and told that they could withdraw from the study whenever they wished. Those patients who provided informed consent completed the questionnaires once during the treatment follow-up period. This study followed the recommendations of the Declaration of Helsinki, and was approved by the Ethics Committee of the Hospital of Navarra.

\section{Statistical analysis}

Sample characteristics and the scores of the QoL and the functional evaluations were described using frequencies and percentages for the categorical variables, and means and standard deviations for the continuous ones.

Inter-group comparisons were made on the basis of surgical modality (LAR versus APR). Prior to that, differences between these two groups were studied for those variables that were considered to possibly influence the QoL scores: age, KPS (Mann-Whitney U tests), gender, the presence of limiting comorbidity, civil status, tumour location, having received adjuvant chemotherapy, and having been in the hospital as an in-patient due to treatment toxicity (Chi square). As no significant differences were found for any of these variables, the QLQ-C30 and QLQ-CR29 scores were compared for the two surgical modality groups. For the body image area, the aforementioned comparisons were also carried out stratifying the sample by sex.

LAR patients were selected and their scores in the EORTC questionnaires (Mann-Whitney U) and the functional evaluation (mean number of bowel movements in 24 hours, degree of faecal incontinence and combined score of bowel function, with Mann-Whitney U; Chi square for the other ten parameters) compared for subgroups based on having or not a reservoir, a temporary ileostomy, and the distance of the anastomosis to the anal verge (2-5/6$12 \mathrm{~cm}) . A p=0.05$ significance level was used for statistical comparisons.

\section{RESULTS}

Eighty-four patients from 90 candidates were evaluated between June 2008 and November 2009. The reasons for not filling in the questionnaires were administrative failure ( 4 cases) and patient refusal ( 2 cases). All patients had received surgery between May 2001 and December 2007. Mean follow-up period was 48.7 months. The sociodemographic and clinical characteristics of the patients are presented in table II.

The QoL scores in the EORTC questionnaires for the sample as a whole were generally high (Tables III and IV). Minor alterations (20-30 points) were detected for global QoL, sleep disturbance and constipation, and stool frequency, anxiety and faecal incontinence. Moderate limitations (> 30 points) were observed for urinary frequency, flatulence, impotence, and the sexual function of men and women. Body image showed high scores (mean 87.3). The functional evaluation and degree of faecal incontinence scores for LAR patients are presented in table V.

Although LAR patients had a higher stool frequency than their APR counterparts, no other differences were found between the two surgery-based groups (tables III and IV). No differences in body image were found amongst patients with stoma or anastomosis when comparing gender-based groups. LAR patients with a reservoir had better emotional functioning $(p=0.04)$ and higher faecal incontinence $(p=0.03)$, whereas those patients with a lower anastomosis had higher faecal incontinence $(p=0.02)$. No differences were found in the comparisons undertaken with 
Table III. Quality of life scores on the EORTC QLQ-C30 general questionnaire

\begin{tabular}{lllll}
\hline Areas & General sample & LAR & APR & $p$ \\
\hline Physical (1) & $85.3(17.6)$ & $87.8(14.1)$ & $78.4(23.8)$ & 0.17 \\
Role (1) & $84.9(24.5)$ & $87.2(20.3)$ & $78.3(33.4)$ & 0.43 \\
Emotional (1) & $83.7(23.1)$ & $81.8(25.4)$ & $88.5(15.5)$ & 0.48 \\
Cognitive (1) & $88.1(20.4)$ & $87.8(21.2)$ & $88.9(18.5)$ & 0.87 \\
Social (1) & $83.3(26.4)$ & $83.9(27.9)$ & $81.7(22.3)$ & 0.36 \\
Global (1) & $71.2(27.2)$ & $70.9(28.0)$ & $71.8(25.7)$ & 0.98 \\
Fatigue (2) & $17.4(24.7)$ & $15.3(23.1)$ & $22.7(28.2)$ & 0.39 \\
Nausea/ & & & & \\
Vomiting (2) & $4.8(17.6)$ & $5.2(17.5)$ & $3.9(18.2)$ & 0.44 \\
Pain (2) & $19.6(25.6)$ & $17.9(25.5)$ & $23.8(26.1)$ & 0.27 \\
Dyspnea (2) & $3.4(12.7)$ & $2.9(11.4)$ & $4.7(15.9)$ & 0.69 \\
Sleep dist. (2) & $24.8(31.1)$ & $25.1(31.8)$ & $23.8(30.1)$ & 0.95 \\
Appetite loss (2) & $12.6(28.1)$ & $13.7(29.7)$ & $9.5(26.1)$ & 0.49 \\
Constipation (2) & $25.1(31.6)$ & $26.8(33.9)$ & $20.6(24.7)$ & 0.76 \\
Diarrhea (2) & $18.6(25.6)$ & $21.4(27.3)$ & $11.1(19.2)$ & 0.14 \\
Financial (2) & $10.9(24.6)$ & $11.5(25.8)$ & $9.5(21.5)$ & 0.97 \\
& & & & \\
\hline
\end{tabular}

Mean and standard deviation (in brackets) of the QL areas in the general questionnaire for the global sample, and for the LAR and APR groups. The scores on the functional scales (1) range from 0 to 100 (higher values indicating better functioning). The symptom scales and items (2) range from 0 to 100 (higher values indicating worse symptoms); p: level of significance (Mann-Whitney $U$ test) in the comparison between the two surgical modalities. the functional evaluation areas. No differences in QoL or functional evaluation were found between patients with or without a temporary ileostomy. To better understand the results regarding the effect of having a reservoir and anastomosis height on faecal incontinence, the variance contribution of these two predictor variables and of having a temporary ileostomy was obtained using the partition of the sums of squares of the variance analysis table. This analysis showed that the three variables explained $13.0 \%$ of the variability, with anastomosis height providing the highest contribution $(4.7 \%)$. Furthermore, although all these variables were significant in the univariate models, none of them were significant in the multivariate model, thereby suggesting the presence of an association between these three covariates that could explain the apparently contradictory results obtained as regards the association between having a reservoir and higher faecal incontinence.

\section{DISCUSSION}

The main results of the present study are that QoL scores of advanced RC patients in the follow-up period after receiving a combined treatment are satisfactory, with minor or moderate limitations in a few specific disease

Table IV. Quality of Life scores on the EORTC QLQ-BR29

\begin{tabular}{|c|c|c|c|c|}
\hline Areas & General sample & $L A R$ & $A P R$ & $p$ \\
\hline Urinary frequency (2) & $38.6(26.8)$ & $38.4(27.3)$ & $39.2(26.1)$ & 0.92 \\
\hline Blood and mucus in stool (2) & $4.4(9.9)$ & $5.2(11.1)$ & $2.4(5.9)$ & 0.41 \\
\hline Body image (1) & $87.3(19.6)$ & $85.4(21.8)$ & $92.1(11.7)$ & 0.49 \\
\hline Urinary incontinence (2) & $14.9(22.0)$ & $12.5(19.7)$ & $21.7(27.1)$ & 0.15 \\
\hline Buttock pain (2) & $16.5(25.1)$ & $16.7(24.6)$ & $15.9(27.2)$ & 0.76 \\
\hline Bloated feeling (2) & $15.4(26.4)$ & $15.2(25.5)$ & $15.8(29.1)$ & 0.92 \\
\hline Dry mouth (2) & $18.6(28.8)$ & $15.5(26.9)$ & $26.9(32.6)$ & 0.13 \\
\hline Hair loss (2) & $4.3(14.7)$ & $4.2(15.6)$ & $4.8(11.9)$ & 0.52 \\
\hline Trouble with taste (2) & $6.9(19.8)$ & $4.8(14.8)$ & $12.7(28.2)$ & 0.28 \\
\hline Anxiety (1) & $78.5(28.1)$ & $79.4(28.3)$ & $79.2(28.2)$ & 0.54 \\
\hline$\star \star$ Embarrassed by bowel movement (2) & $10.5(26.9)$ & $12.3(30.2)$ & $6.3(17.1)$ & 0.72 \\
\hline Stoma care problems (2) & $11.1(21.9)$ & $22.2(38.5)$ & $9.3(19.1)$ & 0.54 \\
\hline Impotence (2) & $62.5(44.8)$ & $70.0(40.5)$ & $40.0(51.6)$ & 0.11 \\
\hline Dyspareunia (2) & $17.3(32.1)$ & $21.1(35.5)$ & $5.6(13.6)$ & 0.39 \\
\hline Sexual function men (1) & $24.6(35.9)$ & $31.2(35.4)$ & $36.1(38.8)$ & 0.69 \\
\hline Sexual function women (1) & $4.8(10.8)$ & $3.5(10.5)$ & $4.8(12.6)$ & 0.79 \\
\hline
\end{tabular}

Mean and standard deviation (in brackets) of the QL areas of the colorectal QLQ-CR29 questionnaire for the global sample and for the low abdominal resection (LAR) and abdominoperineal resection (APR) groups. The scores on the functional scales (1) range from 0 to 100 (higher values indicating better functioning). The symptom scales and items (2) range from 0 to 100 (higher values indicating worse symptoms); p: level of significance (Mann-Whitney $U$ test) in the comparison between the two surgical modalities. ***Each of these areas contains different questions for patients with and without stoma that can be compared. 
Table V. Functional evaluation in low anterior resection (LAR) patients

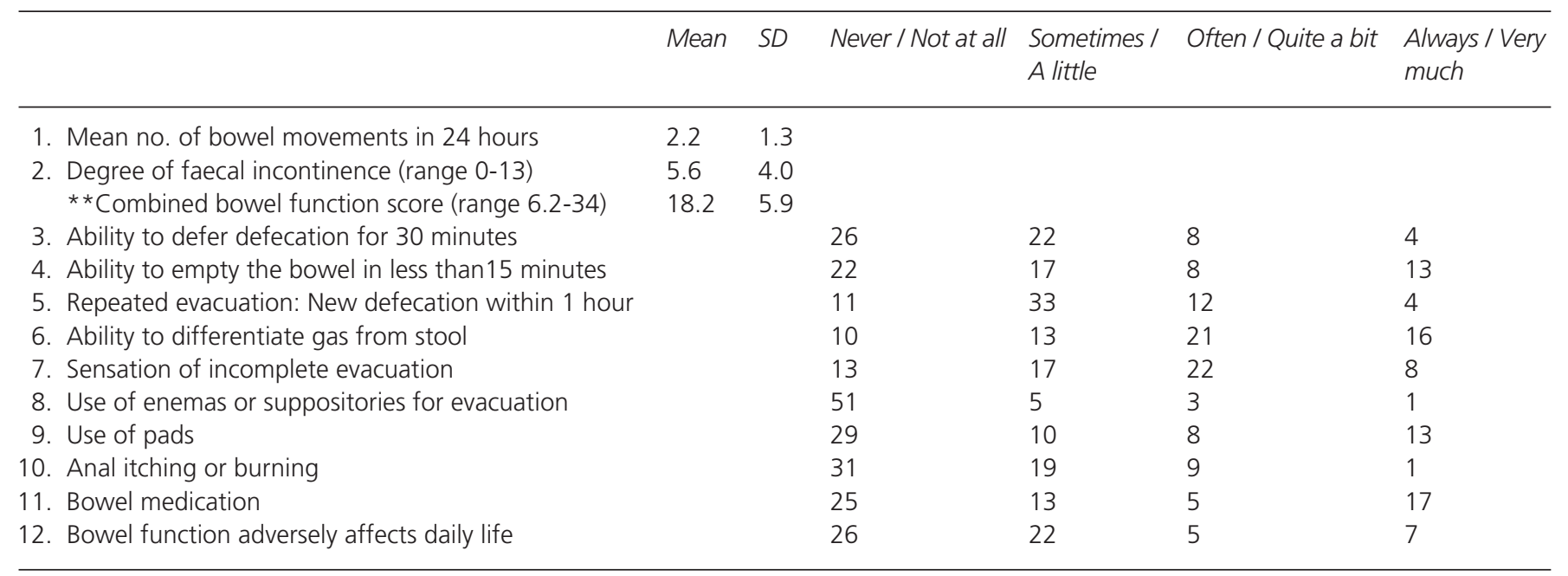

Degree of faecal incontinence: A lower score indicates lower incontinence. Combined bowel function score: A lower score indicates better function. Areas 3 to 12 : Frequencies in the categories of these areas.

and treatment related areas, and in sexuality and emotional dimensions. LAR patients had a higher stool frequency than APR patients with no differences in body image between these two groups. LAR patients with a lower anastomosis had higher faecal incontinence whereas those with a reservoir had better emotional functioning and higher faecal incontinence

The QLQ-C30 scores are in line with the scores found in a Spanish study with RC patients (20), the baseline scores for colorectal cancer patients (stages I-IV) presented in the EORTC Reference Values Manual, and are also very similar to the general population (21). Limitations in the global QoL have been found in other studies carried out by our group (22). The QoL scores in our sample are rather similar to those found in other follow-up studies with RC patients performed with the QLQ-C30 and the QLQ-CR29 $(23,24)$.

Most follow-up studies with RC have administered the previous version of the EORTC colorectal module (QLQCR38). As noted previously, not all areas of the two EORTC questionnaires (QLQ-CR38, QLQ-CR29) can be compared. Despite this, different studies have found similar results to ours in most of the related areas of the two colorectal modules, especially Pucciarelli et al. (25); Allal et al. (26), and Tiv et al. (27), who also administered combined treatments. Other studies have also shown rather similar scores $(9,28,29)$.

Our low scores in the sexual functioning and impotence areas are in line with those reported by Hendren et al. (30) for patients in the follow-up period after undergoing $\mathrm{RC}$ surgery. In contrast, however, these authors found that the type of surgical procedure was associated with sexual activity. The low dyspareunia levels found in our sample could be related to the patients' low level of sexual functioning, something necessary to assess if patients feel pain. Scores in the functional evaluation of LAR patients indicate that patients have a good functioning and are in line with the QoL results and with other studies (31).

The few QoL differences between LAR and APR patients are in line with other studies. We would like to note that the previous version of the colorectal module, QLQCR38, which has been applied in most studies performed with the EORTC instruments, does not allow stool frequency between the two surgical modalities to be compared, thereby possibly preventing results similar to ours in this dimension from being found. Furthermore, QLQ-CR29 allows more comparisons between surgical modalities to be carried out in specific areas than the QLQ-CR38, thereby supporting our conclusion that there are few differences between two surgical modalities studied.

In a meta-analysis of 11 studies (eight of which applied the EORTC instruments, and a further eight of which were performed in the follow-up period), Cornish et al. (5) found no differences in general QoL between APR or LAR, although few differences were detected in specific areas. Furthermore, and similar to the results of our study, these authors found no differences in body image, although differences in this area were found in some of the individual studies $(3,4,9)$. The studies included, and also a recent research (32), have shown a few more differences between surgery modalities than in our study $(3,4,9,33)$. The fact that our patients had a longer followup period than in most of the above-mentioned studies may have led to our detection of an even lower number of differences as the patients in our study may have had more time to adapt to their situation.

In accordance with our results, other studies have found worse bowel functioning in patients with lower anastamosis $(3,6,34)$. Thus, Otto et al. (8) and Guren et al. (9) reported a higher incontinence in lower anastomosis patients. 
Few differences were found between groups of patients with and without a reservoir in the follow-up period, in accordance with other studies $(9,35)$. However, as discussed above, greater differences could have been present at an earlier stage after completion of treatment due to the lower amount of time available for functional and psychological adaptation. In this sense, Sailer et al. (28) found differences in favour of the pouch reconstruction in the early months after surgery but that these differences tended to disappear after one year, the period of time for which we have compared the treatment modalities.

The few differences we have found between patients with and without reservoir could be understood as adequate in the emotional functioning area, showing a better emotional adaptation in the patients with reservoir, but contrary to what we might have expected, and found by other authors, in faecal incontinence (36). In our case the results of the comparisons could have been influenced by the attitude of the anastomosis, which we found might be a more important determinant of faecal incontinence than the presence of a reservoir.

Some of the key points of the present study are that the characteristics of the study sample are representative of the RC patients treated at the Complejo Hospitalario de Navarra. The questionnaire QLQ-CR29 may have helped to get more solid conclusions. Maybe the study could have favoured of a pre-treatment QoL assessment and a higher sample size.

Our findings could indicate there has been a satisfactory adaptation process for patients to their situation along the treatment follow-up period. Besides, Pachler and WilleJørgensen (37), as other authors $(5,6,31)$ and ourselves, concluded by questioning the belief that patients' QoL may improve by avoiding a permanent stoma. In our case, the lack of differences between the biographical and clinical variables for the two surgery groups further support this hypothesis. Information regarding the expected effects of each surgical modality should be offered to patients to help them to adapt better to their situation and, when appropriate, to decide between treatments. It would be useful to perform prospective studies involving long follow-up in order to study the evolution of the differences between surgical modalities, and in which the EORTC QLQ-CR29 is administered.

QL scores and functional evaluation indicated patients had adapted to their disease and treatment. The few differences in QoL found between surgery modalities are in line with other recent studies and in contrast with earlier ones that suggested a lower QoL in APR patients.

\section{ACKNOWLEDGEMENTS}

This study was supported by grant 2443/2009 from the Departamento de Salud del Gobierno de Navarra (Navarre Government Health Department), Spain.

\section{REFERENCES}

1. Trnini Z, Vidacak A, Vrhovac J, Petrov B, Setka V. Quality of life after colorectal cancer surgery in patients from University Clinical Hospital Mostar, Bosnia and Herzegovina. Coll Antropol 2009;33(Supl. 2): $1-5$.

2. Libutti S, Tepper J, Saltz L. Rectal cancer. In: De Vita V, Hellman S, Rosenberg SA, editors. Cancer: Principles and Practice of Oncology. 8th ed. Philadelphia PA: Lippincott, Williams and Wilkins; 2008. p. 1285-98.

3. Camilleri-Brennan J, Steele RJ. Objective assessment of morbidity and quality of life after surgery for low rectal cancer. Colorectal Dis 2002;4:61-6.

4. Sideris L, Zenasni F, Vernerey D, Dauchy S, Lasser P, Pignon JP, et al. Quality of life of patients operated on for low rectal cancer: impact of the type of surgery and patients' characteristics. Dis Colon Rectum 2005;48:2180-91.

5. Cornish JA, Tilney HS, Heriot AG, Lavery IC, Fazio VW, Tekkis PP A meta-analysis of quality of life for abdominoperineal excision of rectum versus anterior resection for rectal cancer. Ann Surg Oncol 2007;14:2056-68.

6. Kasparek MS, Hassan I, Cima RR, Larson DR, Gullerud RE, Wolff BG. Quality of life after coloanal anastomosis and abdominoperineal resection for distal rectal cancers: Sphincter preservation vs quality of life. Colorectal Dis 2011;13:872-7.

7. Gosselink MP, Busschbach JJ, Dijkhuis CM, Stassen LP, Hop WC, Schouten WR. Quality of life after total mesorectal excision for rectal cancer. Colorectal Dis 2006;8:15-22.

8. Otto S, Kroesen AJ, Hotz HG, Buhr HJ, Kruschewski M. Effect of anastomosis level on continence performance and quality of life after colonic J-pouch reconstruction. Dig Dis Sci 2008;53:14-20.

9. Guren MG, Eriksen MT, Wiig JN, Carlsen E, Nesbakken A, Sigurdsson $\mathrm{HK}$, et al. Quality of life and functional outcome following anterior or abdominoperineal resection for rectal cancer. Eur J Surg Oncol 2005;31:735-42.

10. Pucciarelli S, Del Bianco P, Efficace F, Serpentini S, Capirci C, De Paoli A, et al. Patient-reported outcomes after neoadjuvant chemoradiotherapy for rectal cancer: A multicenter prospective observational study. Ann Surg 2011;253:71-7.

11. Bjordal K, de Graeff A, Fayers PM, Hammerlid E, van Pottelsberghe C, Curran D, et al. A 12 country field study of the EORTC QLQ-C30 (version 3.0) and the head and neck cancer specific module (EORTC QLQH\&N35) in head and neck patients. Eur J Cancer 2000;36:1796-807.

12. Whistance RN, Conroy T, Chie W, Costantini A, Sezer O, Koller M, et al. Clinical and psychometric validation of the EORTC QLQ-CR29 questionnaire module to assess health-related quality of life in patients with colorectal cancer. Eur J Cancer 2009;45:3017-26.

13. Arraras JI, Arias F, Tejedor M, Pruja E, Marcos M, Martínez E, et al. The EORTC QLQ-C30 (version 3.0) Quality of Life questionnaire: Validation study for Spain with head and neck cancer patients. Psychooncology 2002;11:249-56.

14. Arraras JI, Suárez J, Arias de la Vega F, Vera R, Asín G, Arrazubi V, et al. The EORTC Quality of Life questionnaire for patients with colorectal cancer: EORTC QLQ-CR29 validation study for Spanish patients. Clin Transl Oncol 2011;13:50-6.

15. Cull A, Sprangers M, Bjordal K, Aaronson N, West K, Bottomley A. EORTC Quality of Life Group translation procedure. 2nd edition. Brussels: EORTC; 2002.

16. Sprangers MAG, Te Velde A, Aaronson NK. The construction and testing of the EORTC Colorectal Cancer-specific Quality of Life Questionnaire Module (QLQ-CR38). Eur J Cancer 1999;35:238-47.

17. Hallböök $O$, Nystrom PO, Sjödahl R. Physiologic characteristics of straight and colonic J-pouch anastomoses after rectal excision for cancer. Dis Colon Rectum 1997;40:332-8.

18. Machado M, Nygren J, Goldman S, Ljungqvist O. Similar outcome after colonic pouch and side-to-end anastomosis in low anterior resection for rectal cancer: A prospective randomized trial. Ann Surg 2003; 238: 214-20.

19. Karnofsky DA, Burchenal JH. The clinical evaluation of chemotherapeutic agents in cancer. In: McLeod CM, editor. Evaluation of chemotherapeutic agents. New York: Colombia University; 1948. p. 199-205. 
20. Sánchez R, Alexander-Sierra F, Oliveros R. Relationship between quality of life and clinical status in patients with gastrointestinal cancer. Rev Esp Enferm Dig 2012;104:584-91

21. Scott N, Fayers P, Aaronson N, Bottomley A, De Graeff A, Groenvold M, et al. EORTC QLQ-C30. Reference Values. Brussels: EORTC, 2008.

22. Arraras JI, Arias de la Vega F, Vera R, Manterola A, Martínez M, Villafranca E, et al. Quality of life assessment through the EORTC questionnaires of locally advanced rectal cancer patients treated with preoperative chemo-radiotherapy. Clin Transl Oncol 2006;8:423-9.

23. Vaughan-Shaw PG, King AT, Cheung T, Beck NE, Knight JS, Nichols $\mathrm{PH}$, et al. Early experience with laparoscopic extralevator abdominoperineal excision within an enhanced recovery setting: analysis of short-term outcomes and quality of life. Ann R Coll Surg Engl 2011;93:451-9.

24. Kripp M, Wieneke J, Kienle P, Welzel G, Brade J, Horisberger K, et al. Intensified neoadjuvant chemoradiotherapy in locally advanced rectal cancer -impact on long-term quality of life. Eur J Surg Oncol 2012;38:472-7.

25. Pucciarelli S, Del Bianco P, Efficace F, Toppan P, Serpentini S, Friso ML, et al. Health-related quality of life, faecal continence and bowel function in rectal cancer patients after chemoradiotherapy followed by radical surgery. Support Care Cancer 2010;18:601-8.

26. Allal AS, Gervaz P, Gertsch P, Bernier J, Roth AD, Morel P, et al. Assessment of quality of life in patients with rectal cancer treated by preoperative radiotherapy: A longitudinal prospective study. Int J Radiat Oncol Biol Phys 2005; 15:1129-35.

27. Tiv M, Puyraveau M, Mineur L, Calais G, Maingon P, Bardet E, et al. Long-term quality of life in patients with rectal cancer treated with preoperative (chemo)-radiotherapy within a randomized trial. Cancer Radiother 2010;14:530-4.

28. Sailer M, Fuchs KH, Fein M, Thiede A. Randomized clinical trial comparing quality of life after straight and pouch coloanal reconstruction. Br J Surg 2002;89:1108-17.
29. Grumann MM, Noack EM, Hoffmann IA, Schlag PM. Comparison of quality of life in patients undergoing abdominoperineal extirpation or anterior resection for rectal cancer. Ann Surg 2001;233:149-56.

30. Hendren SK, O'Connor BI, Liu M, Asano T, Cohen Z, Swallow CJ, et al. Prevalence of male and female sexual dysfunction is high following surgery for rectal cancer. Ann Surg 2005;242:212-23.

31. How P, Stelzner S, Branagan G, Bundy K, Chandrakumaran K, Heald RJ, et al. Comparative quality of life in patients following abdominoperineal excision and low anterior resection for low rectal cancer. Dis Colon Rectum 2012;55:400-6.

32. Peng J, Shi D, Goodman KA, Goldstein D, Xiao C, Guan Z, et al. Early results of quality of life for curatively treated rectal cancers in Chinese patients with EORTC QLQ-CR29. Radiat Oncol 2011;12:93.

33. Rauch P, Miny J, Conroy T, Neyton L, Guillemin F. Quality of life among disease-free survivors of rectal cancer. J Clin Oncol 2004; 22:354-60.

34. Engel J, Kerr J, Schlesinger-Raab A, Eckel R, Sauer H, Hölzel D. Quality of life in rectal cancer patients: A four-year prospective study. Ann Surg 2003;238:203-13.

35. Doeksen A, Bakx R, Vincent A, van Tets WF, Sprangers MA, Gerhards $\mathrm{MF}$, et al. J-pouch vs side-to-end coloanal anastomosis after preoperative radiotherapy and total mesorectal excision for rectal cancer: A multicentre randomized trial. Colorectal Dis 2012;14:705-13.

36. Fürst A, Burghofer K, Hutzel L, Jauch KW. Neorectal reservoir is not the functional principle of the colonic J-pouch: The volume of a short colonic J-pouch does not differ from a straight coloanal anastomosis. Dis Colon Rectum 2002;45:660-7.

37. Pachler J, Wille-Jørgensen P. Quality of life after rectal resection for cancer, with or without permanent colostomy. Cochrane Database Syst Rev 2005;18:CD004323. 